\title{
INFLUÊNCIA DO DIAFRAGMA NO TRATAMENTO PRESERVATIVO DE DUAS ESPÉCIES DE BAMBU POR SUBSTITUIÇÃO DE SEIVA
}

\author{
INFLUENCE OF DIAPHRAGM IN THE PRESERVATIVE TREATMENT OF TWO BAMBOO \\ SPECIES BY SAP DISPLACEMENT METHOD
}

\author{
Rogy Frigeri Tiburtino ${ }^{1}$ Juarez Benigno Paes ${ }^{2}$ Graziela Baptista Vidaurre ${ }^{3}$ \\ Marina Donária Chaves Arantes ${ }^{4}$ Rafael Amorim Rosa ${ }^{5}$
}

\begin{abstract}
RESUMO
Esta pesquisa teve como objetivo avaliar a influência da remoção do diafragma na qualidade do tratamento preservativo de colmos de bambu das espécies Dendrocalamus giganteus e Bambusa vulgaris submetidos ao método de substituição de seiva. Os bambus foram colhidos em touceiras em Alegre e Jerônimo Monteiro, Sul do Espírito Santo. As hastes foram secionadas em colmos de 2,0 m de comprimento. Uma parte dos colmos teve o diafragma removido, enquanto na outra foram mantidos intactos. Os colmos foram tratados em soluções de 1 e $3 \%$ de ingredientes ativos (i.a.) de borato de cobre cromatado (CCB). Os colmos foram mantidos na solução por 5, 10 e 15 dias. Foi avaliada a qualidade do tratamento preservativo por meio da penetração e da retenção do CCB nos colmos. Houve maior penetração de cobre e boro na base dos colmos, a remoção do diafragma proporcionou maior movimentação da solução nos colmos e a retenção obtida ficou, para a maioria das condições testadas, abaixo daquela recomendada pelas normas brasileiras para o tratamento preservativo da madeira.
\end{abstract}

Palavras-chave: qualidade do tratamento; Dendrocalamus giganteus; Bambusa vulgaris.

\begin{abstract}
This research aimed to evaluate the influence of removing the diaphragm on the quality of the preservative treatment of bamboo culms of Dendrocalamus giganteus and Bambusa vulgaris submitted to sap displacement method. The bamboos were collected in clumps in Alegre and Jerônimo Monteiro, south of Espírito Santo state, Brazil. The stems were sectioned in culms of $2.0 \mathrm{~m}$ in length. One part of the culms had the diaphragm removed while in the other they were kept unbroken. The culms were treated in soutions of $1 \%$ and $3 \%$ of active ingredients (a.i.) of copper, chromated copper borate (CCB). The culms were kept in the solution for 5, 10 and 15 days. The preservative treatment quality was evaluated by means of penetration and retention of CCB in culms. There was greater penetration of copper and boron at the base of the culms, the removing of the diaphragm provided greater movement of the solution in the
\end{abstract}

1 Engenheiro Florestal, Msc., Programa de Pós Graduação em Ciências Florestais, Universidade Federal do Espírito Santo, Av. Governador Lindemberg, 316, Centro, CEP 29550-000, Jerônimo Monteiro (ES), Brasil. rogyfrigeri@hotmail.com

2 Engenheiro Florestal, Dr., Professor do Departamento de Ciências Florestais e da Madeira, Universidade Federal do Espírito Santo, Av. Governador Lindemberg, 316, Centro, CEP 29550-000, Jerônimo Monteiro (ES), Brasil. jbp2@uol.com.br

3 Engenheiro Florestal, Dr., Professora do Departamento de Ciências Florestais e da Madeira, Universidade Federal do Espírito Santo, Av. Governador Lindemberg, 316, Centro, CEP 29550-000, Jerônimo Monteiro (ES), Brasil. grazividaurre@gmail.com

4 Engenheiro Florestal, Dr., Professora do Departamento de Ciências Florestais e da Madeira, Universidade Federal do Espírito Santo, Av. Governador Lindemberg, 316, Centro, CEP 29550-000, Jerônimo Monteiro (ES), Brasil. mdonariac@hotmail.com

5 Engenheiro Industrial Madeireiro, Msc., Programa de Pós-Graduação em Ciências Florestais, Universidade Federal do Espírito Santo, Caixa Postal 16, CEP 29550-000, Jerônimo Monteiro (ES), Brasil. rafaelrosa.001@gmail.com

Recebido para publicação em 12/02/2014 e aceito em 20/11/2014 
culms and the retention was, for most treatment conditions, below than the one recommended by Brazilian standards for wood preservative treatment.

Keywords: treatment quality; Dendrocalamus giganteus; Bambusa vulgaris.

\section{INTRODUÇÃO}

Após anos de exploração, as espécies arbóreas nativas de boa durabilidade natural se tornaram escassas em diversas regiões brasileiras (FARIAS SOBRINHO et al., 2005). Por causa dessa situação, a procura por outros materiais de fontes renováveis com características físicas e mecânicas semelhantes às da madeira é cada vez maior, e o bambu surge como alternativa a esses materiais e vem sendo utilizado para diversos fins (PAES et al., 2009). Porém, por possuir elevado teor de amido em sua constituição, são suscetíveis ao ataque de organismos xilófagos, principalmente do coleóptero Dinoderus minutus, que causa danos na estrutura do colmo (DA SILVA, 2007), necessitando de estudos para melhorar seu aproveitamento em construções diversas.

Para atender aos quesitos de durabilidade, o emprego do bambu em construções permanentes exige que sejam realizados tratamentos em seus colmos, pela introdução de substâncias preservativas em sua estrutura celular (AZZINI; BERALDO, 2001). Assim, a técnica de imunização por meio da substituição da seiva pela solução química preservativa é recomendada para se obter bons níveis de resistência do bambu.

De acordo com Pereira e Beraldo (2008) e "National Missionon Bamboo Applications" NMBA (2012), o tratamento por substituição de seiva, consiste em cortar os colmos em peças de 2,0 a 2,5 m de comprimento, colocando-as em sentido vertical na solução preservativa, a qual penetra nos vasos por ação da capilaridade e por difusão. $\mathrm{O}$ tempo para o tratamento preservativo irá depender da umidade, comprimento dos colmos e condições atmosféricas, geralmente variando de 7 a 15 dias.

O colmo do bambu é composto por nós e entrenós. Na região dos nós existe o diafragma, composto por células interligadas transversalmente com intensa ramificação entre os elementos celulares. Já nos entrenós as células são axialmente orientadas (BERALDO; AZZINI, 2004). Tiburtino (2012) observou que na região dos nós houve uma maior difusão da solução preservativa. Hui e Yang (2010) citaram que a presença do diafragma pode dificultar a ascensão de líquido em colmos submetidos ao tratamento de substituição de seiva.

Segundo Tiburtino (2012), a maior parte das pesquisas sobre o tratamento químico de bambu com o método de substituição de seiva tem empregado o borato de cobre cromatado (CCB), pois este é considerado menos nocivo à saúde do operador e ao ambiente, quando comparado aos produtos que contêm arsênio em sua formulação, como o arseniato de cobre cromatado (CCA).

A maioria dos trabalhos com tratamentos preservativos de bambu não apresentam a preocupação em avaliar a qualidade desse tratamento e a distribuição da solução preservativa ao longo do colmo. Neste sentido, esta pesquisa teve como objetivo avaliar a influência da remoção do diafragma na penetração e retenção do borato de cobre cromatado (CCB) em colmos de bambu das espécies Dendrocalamus giganteus e Bambusa vulgaris submetidos ao método de substituição de seiva.

\section{MATERIAL E MÉTODOS}

\section{Espécies, procedência e coleta dos bambus}

Para a realização deste trabalho foram utilizados bambus das espécies Dendrocalamus giganteus e Bambusa vulgaris provenientes de três touceiras situadas nos municípios de Alegre e Jerônimo Monteiro, sul do Estado do Espírito Santo. Foram retirados bambus que possuíam manchas brancas no colmo, que, segundo Pereira e Beraldo (2008), caracterizam bambus com idade superior a três anos. Para o corte dos colmos foram utilizados motosserra e ferramentas manuais, como foice e facão. $O$ corte foi realizado a $20 \mathrm{~cm}$ do solo e logo acima de um dos nós, conforme indicado por Santos e Lopes (1998).

Depois de colhidos, os colmos foram medidos e seccionados em partes de 2,20 m de comprimento. Antes do tratamento preservativo, foram retirados dois anéis de $5,0 \mathrm{~cm}$ de comprimento na base e no topo dos colmos, ficando os colmos com $2,00 \mathrm{~m}$. A primeira amostra de cada posição foi descartada e a segunda (mais interna) utilizada para avaliação da umidade dos colmos. 


\section{Produto químico e processo de imunização empregado}

Para o tratamento preservativo (imunização) dos bambus foi utilizado um produto hidrossolúvel, fungicida e inseticida à base de borato de cobre cromatado (CCB), que possui boa mobilidade na madeira e que é indicado para a imunização da madeira verde. Foram utilizadas soluções de 1 e $3 \%$ de ingredientes ativos. Antes da imunização, os colmos foram identificados e agrupados de forma que os tratamentos tivessem volumes semelhantes.

O processo de imunização foi realizado sob duas situações: diafragma íntegro e diafragma rompido. Para o rompimento do diafragma foi utilizada uma barra metálica. Durante o procedimento, os colmos foram dispostos verticalmente na solução preservativa por $5 ; 10 \mathrm{ou}$ 15 dias, em tambores de $200 \mathrm{~L}$, ficando submersos ( $0,5 \mathrm{~m}$ da base) na solução e mantidas suas porções aéreas separadas, a fim de proporcionar boa aeração entre os colmos. Para evitar a evaporação da água e o desbalanceamento da solução preservativa, derramaram-se $200 \mathrm{~mL}$ de óleo lubrificante na superfície da solução.

O processo de imunização foi efetuado ao ar livre e em área coberta, sendo diariamente registrada a quantidade de solução absorvida pelos colmos e efetuada a sua reposição, a fim de manter constante o nível inicial de solução nos tambores.

\section{Secagem e amostragem dos colmos tratados}

Ao término do processo, os colmos foram empilhados por 30 dias, em local sombreado e ventilado. Depois de secos, foram retirados discos de $\pm 2,5 \mathrm{~cm}$ de altura, em três posições nos colmos ( $0,5 \mathrm{~m}$ da base, $1,00 \mathrm{~m}$ e 1,90 $\mathrm{m}$ da base) .

Nos discos foi realizada análise química para a determinação da penetração do cobre e do boro, conforme as recomendações da Norma Brasileira Regulamentadora - NBR 6232 da Associação Brasileira de Normas Técnica - ABNT (2013). Em um disco adjacente àquele obtido a $50 \mathrm{~cm}$ da base dos colmos, foram retiradas amostras para a realização da análise de retenção dos ingredientes ativos (i.a.) do CCB.

\section{Análises de penetração e retenção do CCB}

A penetração do boro e cobre na peça tratada foi avaliada por meio de notas, sendo considerada a média da nota atribuída por cinco avaliadores, de acordo com os padrões de penetração descritos por Sales-Campos et al. (2003), sendo a penetração nula (nota $=0)$, penetração vascular $(1)$, penetração parcial irregular (2), penetração parcial periférica (3) e penetração total (4), conforme Figura 1.

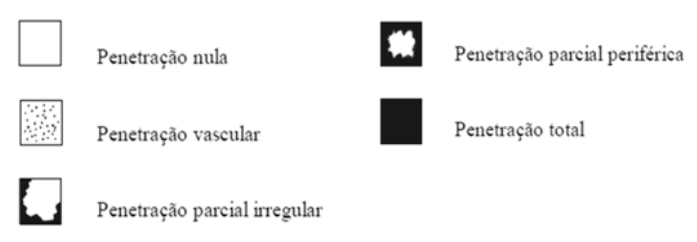

FIGURA 1: Padrões de penetração de preservativos na madeira (SALES-CAMPOS et al., 2003).

FIGURE 1: Penetration patterns of preservatives in wood (SALES-CAMPOS et al., 2003).

A determinação da quantidade de ingredientes ativos retidos nas amostras foi realizada seguindo a metodologia descrita por Wischer, citada por Paes et al. (2005) e Torres et al. (2011). Inicialmente, foi mensurado o volume da amostra de bambu seca em estufa a $103 \pm 2{ }^{\circ} \mathrm{C}$ por 48 horas. Em seguida, essas amostras foram colocadas em mufla para serem incineradas à temperatura de 500 $-550^{\circ} \mathrm{C}$ por 4 horas, até ocorrer a sua transformação em cinzas. Após remover o material da mufla, foram adicionados às cinzas $3 \mathrm{~mL}$ da mistura dos ácidos sulfúrico, perclórico e nítrico, todos concentrados, nas proporções 7:2:1.

Em uma chapa aquecida foi realizada a digestão acelerada da mistura dos ácidos com as cinzas das amostras. O aquecimento ocorreu até que a mistura ficasse límpida, sendo diluídas as soluções ácidas obtidas em água destilada a volumes fixos de $100 \mathrm{~mL}$.

$\mathrm{Na}$ determinação do cobre e do cromo foi empregado o espectrofotômetro de absorção atômica; o boro foi analisado por fotocolorimetria. Para este trabalho, a retenção de $6,5 \mathrm{~kg}$ de i.a. $\mathrm{m}^{-3}$, recomendada pela NBR 9480 (ABNT, 2009), foi considerada como o valor mínimo aceitável.

Com os dados obtidos pela espectrofotometria e pela fotocolorimetria, e com o volume das amostras de bambu, os cálculos de retenção foram efetuados de acordo com a Equação 1, citada por Paes et al. (2005) e Torres et al. (2011).

$$
\mathrm{R}=\frac{\mathrm{F} \times \mathrm{L} \times \mathrm{Fd} \times 10^{-3}}{\mathrm{~V}}
$$


Em que: $\mathrm{R}=$ Retenção do elemento no bambu (kg i.a. $\left.\mathrm{m}^{-3}\right) ; \mathrm{F}=$ Fator estequiométrico empregado para transformação dos elementos químicos para óxidos $\left(\right.$ cobre x 1,2518 $=\mathrm{CuO}$, cromo x 1,9230 $\left.=\mathrm{CrO}_{3}\right) ; \mathrm{L}$ $=$ Leitura obtida do elemento químico $\left(\mathrm{mg} . \mathrm{L}^{-1}\right) ; \mathrm{Fd}$ $=$ Fator de diluição necessário para as leituras dos elementos; e $\mathrm{V}=$ Volume das amostras de bambu secas em estufa utilizadas nas análises $\left(\mathrm{cm}^{3}\right)$.

\section{Análises estatísticas dos dados}

Para a análise da penetração e retenção do preservativo foi empregado um delineamento inteiramente casualizado com arranjo fatorial, a fim de avaliar os efeitos das posições nos colmos, tempo no tratamento preservativo e concentração das soluções de CCB.

Para avaliar a penetração em cada posição nos colmos, foram avaliados os efeitos das posições (três níveis), tempo no tratamento preservativo (três níveis) e concentração das soluções (dois níveis), com quatro repetições para cada situação testada (diafragma íntegro ou rompido). Para esta análise, foram fixados os fatores situação, espécie de bambu e o elemento químico (cobre ou boro).

Para a análise da retenção, o número de amostras foi reduzido após ter sido verificado que houve boa penetração, de modo geral, apenas nas amostras coletadas na posição tomada na base do colmo, tendo sido empregadas duas repetições para cada situação de tratamento, e analisados os efeitos da situação (dois níveis), tempo no tratamento preservativo (três níveis) e concentração das soluções de CCB (dois níveis).

De modo semelhante ao ocorrido para a análise da retenção, o efeito da situação de tratamento (diafragma íntegro ou rompido) na penetração da solução preservativa foi avaliado apenas para a posição da base do colmo. Tendo sido fixados os fatores espécie de bambu e o elemento químico (cobre ou boro).

Para a análise estatística da penetração dos elementos cobre e boro, os dados foram transformados em raiz (notas $+0,5$ ), como sugerido por Steel e Torrie (1980). Esta transformação foi necessária quando da necessidade de homogeneizar as variâncias. Foi empregado o teste de Tukey, a 5\% de significância, para comparação das médias, para os fatores e interações detectados como significativos pelo teste $\mathrm{F}(\mathrm{p} \leq 0,05)$.

\section{RESULTADOS E DISCUSSÃO}

\section{Umidade dos bambus e absorção da solução de CCB pelos colmos}

A umidade dos colmos de Dendrocalamus giganteus variou de 61,97 a $72,62 \%$ (diafragma íntegro) e de 74,25 a 94,53\% (diafragma rompido), enquanto para o Bambusa vulgaris de 74,25 a 94,53\% (diafragma íntegro) e de 91,96 a 104,04\% (diafragma rompido). De acordo com esses valores, as duas espécies de bambu continham umidade suficiente (superior a 50\%) para serem tratadas pelo método de tratamento empregado, segundo Farias Sobrinho et al. (2005), Paes et al. (2005) e Torres et al. (2011).

As quantidades totais de solução absorvidas para o tratamento preservativo da espécie Dendrocalamus giganteus com diafragma íntegro, para a solução de $1 \%$ de CCB foi de $66,3 \mathrm{~L} \mathrm{e}$, para a solução de 3\%, de 60,4 L. Enquanto para o Bambusa vulgaris foram repostos $18,9 \mathrm{~L}$ para a solução de $1 \%$ e $23,3 \mathrm{~L}$ para a de $3 \%$.

Já no tratamento preservativo com diafragma rompido foram repostos 51,4 e 43,8L para as soluções com concentrações de 1 e $3 \%$, respectivamente. respectivamente, para o Dendrocalamus giganteus, enquanto para o Bambusa vulgaris, as reposições foram de 32,8 e $27,5 \mathrm{~L}$ para as soluções com concentrações de $1 \mathrm{e}$ $3 \%$, respectivamente.

\section{Penetração de cobre e boro nos bambus}

Constam nas Tabelas 1 e 2 os valores médios das notas de penetração do cobre e boro, respectivamente. Observa-se que, de maneira geral, para as condições avaliadas, que as maiores notas para ambos os elementos químicos foram obtidas na base dos colmos, seguidas pelo meio e topo dos colmos.

Ferreira (2010) encontrou resultados semelhantes ao avaliar a penetração do cobre nas regiões da base $(0,55 \mathrm{~m}$ de altura), meio $(1,10 \mathrm{~m}$ de altura) e topo (2,10 m de altura) em colmos de Bambusa vulgaris tratados durante 3, 6 e 9 dias. Não foram encontrados na literatura trabalhos que exibissem resultados de penetração do boro em bambu.

A penetração do cobre em cada situação analisada indicou diferença estatística apenas para o parâmetro posição no colmo para a 
TABELA 1: Penetrações de cobre por situação, espécie, concentração da solução, tempo de tratamento e posição nos colmos tratados.

TABLE 1: Copper penetrations for situation, species, and concentration of solution, treatment time and position in treated culms.

\begin{tabular}{|c|c|c|c|c|c|c|}
\hline \multirow{2}{*}{ Situação } & \multirow{2}{*}{ Espécie } & \multirow{2}{*}{ Conc. $(\%)$} & \multirow{2}{*}{ Tempo (Dias) } & \multicolumn{3}{|c|}{ Penetração por posição nos colmos (Nota) } \\
\hline & & & & Base & Meio & Topo \\
\hline \multirow{12}{*}{$\begin{array}{l}\text { Diafragma } \\
\text { Íntegro }\end{array}$} & \multirow{6}{*}{$\begin{array}{l}\text { Dendrocalamus } \\
\text { giganteus }\end{array}$} & \multirow{3}{*}{1} & 5 & $0,65 \pm 0,19$ & $0,35 \pm 0,10$ & $0,25 \pm 0,19$ \\
\hline & & & 10 & $0,80 \pm 0,16$ & $0,40 \pm 0,36$ & $0,00 \pm 0,00$ \\
\hline & & & 15 & $0,95 \pm 0,19$ & $0,20 \pm 0,28$ & $0,10 \pm 0,11$ \\
\hline & & \multirow{3}{*}{3} & 5 & $0,80 \pm 0,28$ & $0,50 \pm 0,20$ & $0,00 \pm 0,00$ \\
\hline & & & 10 & $1,30 \pm 0,41$ & $0,60 \pm 0,28$ & $0,10 \pm 0,11$ \\
\hline & & & 15 & $0,95 \pm 0,25$ & $0,50 \pm 0,47$ & $0,05 \pm 0,10$ \\
\hline & \multirow{6}{*}{ Bambusa vulgaris } & \multirow{3}{*}{1} & 5 & $0,75 \pm 0,37$ & $0,40 \pm 0,43$ & $0,00 \pm 0,00$ \\
\hline & & & 10 & $1,05 \pm 0,19$ & $0,90 \pm 0,47$ & $0,50 \pm 0,25$ \\
\hline & & & 15 & $1,65 \pm 0,80$ & $1,25 \pm 0,66$ & $0,85 \pm 0,47$ \\
\hline & & \multirow{3}{*}{3} & 5 & $1,45 \pm 0,99$ & $0,65 \pm 0,25$ & $0,15 \pm 0,19$ \\
\hline & & & 10 & $1,40 \pm 0,36$ & $0,85 \pm 0,19$ & $0,40 \pm 0,16$ \\
\hline & & & 15 & $2,05 \pm 0,50$ & $1,15 \pm 0,50$ & $0,50 \pm 0,34$ \\
\hline \multirow{12}{*}{$\begin{array}{l}\text { Diafragma } \\
\text { Rompido }\end{array}$} & \multirow{6}{*}{$\begin{array}{l}\text { Dendrocalamus } \\
\quad \text { Giganteus }\end{array}$} & \multirow{3}{*}{1} & 5 & $1,00 \pm 0,16$ & $0,80 \pm 0,16$ & $0,25 \pm 0,25$ \\
\hline & & & 10 & $1,45 \pm 0,77$ & $1,00 \pm 0,67$ & $0,40 \pm 0,28$ \\
\hline & & & 15 & $1,25 \pm 0,19$ & $0,90 \pm 0,20$ & $0,20 \pm 0,16$ \\
\hline & & \multirow{3}{*}{3} & 5 & $1,95 \pm 1,36$ & $0,90 \pm 0,11$ & $0,35 \pm 0,34$ \\
\hline & & & 10 & $2,95 \pm 1,27$ & $1,05 \pm 0,19$ & $0,55 \pm 0,19$ \\
\hline & & & 15 & $2,30 \pm 0,38$ & $1,30 \pm 0,25$ & $0,95 \pm 0,41$ \\
\hline & \multirow{6}{*}{ Bambusa Vulgaris } & \multirow{3}{*}{1} & 5 & $1,50 \pm 0,52$ & $1,10 \pm 0,25$ & $1,00 \pm 0,32$ \\
\hline & & & 10 & $1,35 \pm 0,95$ & $0,70 \pm 0,38$ & $0,65 \pm 0,44$ \\
\hline & & & 15 & $1,45 \pm 0,64$ & $1,10 \pm 0,47$ & $0,95 \pm 0,55$ \\
\hline & & \multirow{3}{*}{3} & 5 & $1,60 \pm 0,51$ & $1,55 \pm 0,80$ & $1,00 \pm 0,71$ \\
\hline & & & 10 & $2,20 \pm 1,24$ & $1,35 \pm 0,41$ & $1,60 \pm 0,71$ \\
\hline & & & 15 & $2,50 \pm 1,06$ & $2,00 \pm 0,81$ & $1,05 \pm 0,41$ \\
\hline
\end{tabular}

Em que: Conc. $=$ Concentração da solução preservativa.

espécie Dendrocalamus giganteus, no tratamento preservativo com diafragma íntegro. Para o mesmo tratamento, o Bambusa vulgaris exibiu significância entre os parâmetros tempo, posição e entre a interação de segunda ordem (concentração x tempo x posição).

O tratamento preservativo com diafragma rompido obteve no Dendrocalamus giganteus variação entre os parâmetros concentração, tempo e posição no colmo e entre a interação de primeira ordem (concentração x posição). Na espécie Bambusa vulgaris, observou-se diferença significativa apenas entre os parâmetros concentração e posição no colmo.

Para ambas as espécies, a posição da base obteve maior penetração, seguida pela posição do meio e topo dos colmos tratados. O tempo de 15 dias para Bambusa vulgaris propiciou maior penetração do elemento cobre, seguidos pelos tempos de 10 e 5 dias (Tabela 3).

$\mathrm{Na}$ Tabela 4 estão as comparações das médias de penetração do cobre no tratamento preservativo com diafragma rompido. Foi verificado que para o Dendrocalamus giganteus, os tempos de 10 e 15 dias obtiveram as penetrações mais elevadas. A concentração de 3\% propiciou melhores penetrações nas três posições nos colmos.

Nota-se um comportamento semelhante de penetração no Bambusa vulgaris em que as posições da base e meio e a concentração de $3 \%$ alcançaram as 
TABELA 2: Penetrações de boro por situação, espécie, concentração da solução, tempo de tratamento e posição nos colmos tratados.

TABLE 2: Boron penetrations for treatment time, concentration and situation to the studied species.

\begin{tabular}{|c|c|c|c|c|c|c|}
\hline \multirow{2}{*}{ Situação } & \multirow{2}{*}{ Espécie } & \multirow{2}{*}{$\begin{array}{l}\text { Conc. } \\
(\%)\end{array}$} & \multirow{2}{*}{$\begin{array}{l}\text { Tempo } \\
\text { (Dias) }\end{array}$} & \multicolumn{3}{|c|}{ Penetração por posição nos colmos (Nota) } \\
\hline & & & & Base & Meio & Topo \\
\hline \multirow{12}{*}{ Diafragma Íntegro } & \multirow{7}{*}{$\begin{array}{l}\text { Dendrocalamus } \\
\text { giganteus }\end{array}$} & \multirow{3}{*}{1} & 5 & $2,65 \pm 0,34$ & $1,60 \pm 0,58$ & $1,50 \pm 0,62$ \\
\hline & & & 10 & $2,95 \pm 0,50$ & $1,65 \pm 0,61$ & $0,75 \pm 0,37$ \\
\hline & & & 15 & $2,95 \pm 0,34$ & $1,60 \pm 0,32$ & $0,85 \pm 0,41$ \\
\hline & & \multirow{3}{*}{3} & 5 & $2,45 \pm 0,71$ & $1,75 \pm 0,52$ & $0,55 \pm 0,44$ \\
\hline & & & 10 & $3,35 \pm 0,59$ & $2,10 \pm 0,11$ & $1,55 \pm 0,19$ \\
\hline & & & 15 & $3,50 \pm 0,57$ & $1,90 \pm 0,66$ & $0,40 \pm 0,56$ \\
\hline & & \multirow{3}{*}{1} & 5 & $3,55 \pm 0,38$ & $1,40 \pm 0,43$ & $1,10 \pm 0,11$ \\
\hline & \multirow{5}{*}{$\begin{array}{l}\text { Bambusa } \\
\text { vulgaris }\end{array}$} & & 10 & $3,85 \pm 0,19$ & $2,25 \pm 0,75$ & $1,85 \pm 0,44$ \\
\hline & & & 15 & $3,60 \pm 0,46$ & $2,60 \pm 0,73$ & $1,35 \pm 0,25$ \\
\hline & & \multirow{3}{*}{3} & 5 & $3,65 \pm 0,34$ & $1,80 \pm 0,58$ & $1,15 \pm 0,19$ \\
\hline & & & 10 & $3,75 \pm 0,19$ & $1,30 \pm 0,34$ & $1,25 \pm 0,34$ \\
\hline & & & 15 & $3,80 \pm 0,23$ & $2,90 \pm 0,20$ & $1,40 \pm 0,54$ \\
\hline \multirow{12}{*}{$\begin{array}{l}\text { Diafragma } \\
\text { Rompido }\end{array}$} & \multirow{6}{*}{$\begin{array}{l}\text { Dendrocalamus } \\
\text { giganteus }\end{array}$} & \multirow{3}{*}{1} & 5 & $2,70 \pm 0,90$ & $1,15 \pm 0,68$ & $0,80 \pm 0,71$ \\
\hline & & & 10 & $2,10 \pm 0,68$ & $0,95 \pm 0,19$ & $0,70 \pm 0,38$ \\
\hline & & & 15 & $3,05 \pm 0,68$ & $1,65 \pm 0,91$ & $0,85 \pm 0,30$ \\
\hline & & \multirow{3}{*}{3} & 5 & $3,40 \pm 0,71$ & $2,00 \pm 0,71$ & $0,70 \pm 0,57$ \\
\hline & & & 10 & $2,75 \pm 1,01$ & $1,45 \pm 1,57$ & $0,85 \pm 0,80$ \\
\hline & & & 15 & $2,25 \pm 0,85$ & $1,35 \pm 0,98$ & $0,75 \pm 0,19$ \\
\hline & \multirow{6}{*}{$\begin{array}{l}\text { Bambusa } \\
\text { vulgaris }\end{array}$} & \multirow{3}{*}{1} & 5 & $3,35 \pm 0,59$ & $2,00 \pm 0,51$ & $1,70 \pm 0,62$ \\
\hline & & & 10 & $3,55 \pm 0,25$ & $2,95 \pm 0,52$ & $2,15 \pm 0,99$ \\
\hline & & & 15 & $3,55 \pm 0,41$ & $3,15 \pm 0,52$ & $1,75 \pm 1,11$ \\
\hline & & \multirow{3}{*}{3} & 5 & $3,10 \pm 0,77$ & $2,70 \pm 0,50$ & $1,60 \pm 0,23$ \\
\hline & & & 10 & $1,90 \pm 0,25$ & $1,70 \pm 0,62$ & $1,95 \pm 0,98$ \\
\hline & & & 15 & $2,75 \pm 0,66$ & $2,60 \pm 0,83$ & $2,00 \pm 1,04$ \\
\hline
\end{tabular}

Em que: Conc. $=$ Concentração da solução preservativa.

notas mais elevadas, ou seja, melhores penetrações. As penetrações na base e no meio dos colmos das duas espécies foram estatisticamente semelhantes, o que pode ser explicado pelo rompimento do diafragma que proporcionou a movimentação do CCB no interior dos colmos, uma vez que a solução era retida nos diafragmas, e apenas após o preenchimento total dos mesmos, continuava sua ascensão, conforme afirmado por Hui e Yang (2010) e observado por Tiburtino (2012).

Para a penetração do boro nos bambus tratados com diafragma íntegro, foi observada diferença para a posição e interação entre concentração $\mathrm{x}$ tempo para o Dendrocalamus giganteus. O Bambusa vulgaris obteve variação para o tempo e posição e para as interações concentração $\mathrm{x}$ tempo e tempo x posição. As análises de variância para os bambus com diafragma rompido exibiram diferenças estatísticas para a posição no colmo no Dendrocalamus giganteus. No Bambusa vulgaris observou-se diferença para a concentração e posição no colmo, e para a interação concentração $\mathrm{x}$ tempo.

As médias para a penetração do boro em colmos submetidos ao método com diafragma íntegro estão apresentadas na Tabela 5. No Dendrocalamus giganteus a posição da base obteve maior penetração com relação às posições do meio e do topo. Os colmos da mesma espécie tratados em solução com concentração de $1 \%$ não sofreram influência do tempo, mas nos colmos tratados com $3 \%$ foi verificado que a penetração no tempo de 10 dias foi maior. 
TABELA 3: Penetrações de cobre para tempo e posição no colmo para espécies estudadas submetidas ao tratamento preservativo com diafragma íntegro.

TABLE 3: Copper penetrations for time and position in the culms for the studied species submitted to preservative treatment with diaphragm integrity.

\begin{tabular}{cc}
\hline \multicolumn{3}{c}{ Diafragma Íntegro } \\
\hline Posição & Pendrocalamus giganteus \\
\hline Base & $1,18 \mathrm{a}$ \\
Meio & $0,95 \mathrm{~b}$ \\
Topo & $0,76 \mathrm{c}$ \\
\hline & \\
\hline Posição & Bambusa vulgaris \\
\hline Base & Penetração (Nota) \\
Meio & $1,35 \mathrm{a}$ \\
Topo & $1,15 \mathrm{~b}$ \\
\hline Tempo (Dias) & $0,93 \mathrm{c}$ \\
\hline 5 & Penetração (Nota) \\
10 & $0,99 \mathrm{c}$ \\
15 & $1,15 \mathrm{~b}$ \\
& $1,30 \mathrm{a}$
\end{tabular}

Em que: As médias seguidas da mesma letra, para cada parâmetro, não diferem estatisticamente pelo teste de Tukey $(\mathrm{p}>0,05)$.

TABELA 4: Penetrações de cobre para concentração, tempo e posição no colmo nas espécies estudadas submetidas ao tratamento preservativo com diafragma rompido.

TABLE 4: Copper penetrations for concentration, time and position in the culms for studied species submitted to preservative treatment with ruptured diaphragm.

\begin{tabular}{|c|c|c|c|}
\hline \multicolumn{4}{|c|}{ Diafragma Rompido } \\
\hline \multicolumn{4}{|c|}{ Dendrocalamus giganteus } \\
\hline Tempo (Dias) & \multicolumn{3}{|c|}{ Penetração (Nota) } \\
\hline 5 & \multicolumn{3}{|c|}{$1,14 \mathrm{~b}$} \\
\hline 10 & \multicolumn{3}{|c|}{$1,27 \mathrm{a}$} \\
\hline 15 & \multicolumn{3}{|c|}{$1,25 \mathrm{ab}$} \\
\hline \multirow{2}{*}{ Concentração (\%) } & \multicolumn{3}{|c|}{ Posição/Penetração (Nota) } \\
\hline & Base & Meio & Topo \\
\hline 1 & $1,30 \mathrm{Ab}$ & $1,17 \mathrm{Aa}$ & $0,87 \mathrm{Bb}$ \\
\hline 3 & $1,67 \mathrm{Aa}$ & $1,25 \mathrm{Ba}$ & $1,04 \mathrm{Ca}$ \\
\hline \multicolumn{4}{|c|}{ Bambusa vulgaris } \\
\hline Concentração (\%) & \multicolumn{3}{|c|}{ Penetração (Nota) } \\
\hline 1 & \multicolumn{3}{|c|}{$1,24 \mathrm{~b}$} \\
\hline 3 & \multicolumn{3}{|c|}{$1,43 \mathrm{a}$} \\
\hline Posição & \multicolumn{3}{|c|}{ Penetração (Nota) } \\
\hline Base & \multicolumn{3}{|c|}{$1,48 \mathrm{a}$} \\
\hline Meio & \multicolumn{3}{|l|}{$1,32 \mathrm{ab}$} \\
\hline Topo & \multicolumn{3}{|l|}{$1,22 \mathrm{~b}$} \\
\hline
\end{tabular}

Em que: As médias seguidas da mesma letra, maiúscula na horizontal, ou minúscula na vertical, para cada parâmetro, não diferem estatisticamente pelo teste de Tukey $(\mathrm{p}>0,05)$. 
TABELA 5: Penetrações de boro para concentração, tempo e posição no colmo para espécies estudadas submetidas ao tratamento preservativo com diafragma íntegro.

TABLE 5: Boron penetrations for concentration, time and position in the culms for studied species submitted to preservative treatment with diaphragm integrity.

\begin{tabular}{|c|c|c|c|}
\hline \multicolumn{4}{|c|}{ Diafragma Íntegro } \\
\hline \multicolumn{4}{|c|}{ Dendrocalamus giganteus } \\
\hline Posição & \multicolumn{3}{|c|}{ Penetração (Nota) } \\
\hline Base & \multicolumn{3}{|c|}{$1,85 \mathrm{a}$} \\
\hline Meio & \multicolumn{3}{|c|}{$1,50 \mathrm{~b}$} \\
\hline Topo & \multicolumn{3}{|c|}{$1,16 \mathrm{c}$} \\
\hline \multirow{2}{*}{ Concentração (\%) } & \multicolumn{3}{|c|}{ Tempo (Dias)/Penetração (Nota) } \\
\hline & 5 & 10 & 15 \\
\hline 1 & $1,53 \mathrm{Aa}$ & $1,47 \mathrm{Ab}$ & $1,48 \mathrm{Aa}$ \\
\hline 3 & $1,40 \mathrm{Ba}$ & $1,67 \mathrm{Aa}$ & $1,49 \mathrm{Ba}$ \\
\hline \multicolumn{4}{|c|}{ Bambusa vulgaris } \\
\hline \multirow{2}{*}{ Concentração (\%) } & \multicolumn{3}{|c|}{ Tempo (Dias)/Penetração (Nota) } \\
\hline & 5 & 10 & 15 \\
\hline 1 & $1,55 \mathrm{Ba}$ & $1,75 \mathrm{Aa}$ & $1,71 \mathrm{Aa}$ \\
\hline 3 & $1,60 \mathrm{Ba}$ & $1,57 \mathrm{Bb}$ & $1,76 \mathrm{Aa}$ \\
\hline \multirow{2}{*}{ Tempo (Dias) } & \multicolumn{3}{|c|}{ Posição/Penetração (Nota) } \\
\hline & Base & Meio & Topo \\
\hline 5 & $2,02 \mathrm{Aa}$ & $1,44 \mathrm{Bb}$ & $1,27 \mathrm{Ca}$ \\
\hline 10 & $2,07 \mathrm{Aa}$ & $1,50 \mathrm{Bb}$ & $1,42 \mathrm{Ba}$ \\
\hline 15 & $2,04 \mathrm{Aa}$ & $1,80 \mathrm{Ba}$ & $1,36 \mathrm{Ca}$ \\
\hline
\end{tabular}

Em que: As médias seguidas da mesma letra, maiúscula na horizontal, ou minúscula na vertical, para cada parâmetro, não diferem estatisticamente pelo teste de Tukey $(\mathrm{p}>0,05)$.

Para o Bambusa vulgaris, os tempos de 10 e 15 dias proporcionaram penetrações similares na concentração de $1 \%$. Foi observado que os colmos tratados com 3\% em tempo de 15 dias obtiveram melhores resultados. Independentemente do tempo, a posição da base exibiu penetração mais intensa de boro; a posição do meio indicou diferença estatística sendo superior para o tempo de 15 dias.

Para a penetração do boro no tratamento preservativo com diafragma rompido, a posição da base, para ambas as espécies, proporcionou os melhores resultados. $\mathrm{Na}$ interação concentração $\mathrm{x}$ posição, houve diferença significativa somente na posição do meio, em que a penetração na concentração de $1 \%$ foi superior, quando comparada a de $3 \%$ (Tabela 6 ).

Verificou-se, de modo geral, que os maiores valores de penetração do cobre e boro foram obtidos, principalmente, nas amostras retiradas na base $(0,5$ $\mathrm{m}$ de altura) dos colmos para ambas as espécies e condições avaliadas. Assim, a comparação entre as condições de tratamento (diafragma íntegro ou rompido) foi realizada apenas para as amostras provenientes da base dos colmos.

Houve diferenças significativas para o Dendrocalamus giganteus entre o fator tempo e a interação entre a concentração $\mathrm{x}$ situação de tratamento para o cobre e para a interação tempo $\mathrm{x}$ situação de tratamento para o boro. Para o Bambusa vulgaris foram observadas diferenças estatísticas entre o fator concentração para o cobre e entre as interações tempo x concentração; tempo x situação de tratamento; e concentração $\mathrm{x}$ situação de tratamento para o boro. Os fatores e as interações, depois do desdobramento, constam nas Tabelas $7 \mathrm{e}$ 8 , para o cobre e boro, respectivamente.

Nota-se para a penetração do cobre (Tabela 7), que o efeito da condição de tratamento foi significativo apenas para o Dendrocalamus giganteus, em que os colmos que tiveram os 
diafragmas rompidos obtiveram uma maior penetração, com destaque para a solução mais concentrada. Os efeitos dos demais fatores foram discutidos anteriormente.
Para a penetração de boro (Tabela 8), tanto no Dendrocalamus giganteus quanto no Bambusa vulgaris, não se observaram diferenças marcantes entre os valores obtidos para os colmos tratados com

TABELA 6: Penetrações de boro para concentração e posição no colmo para espécies estudadas submetidas ao tratamento com diafragma íntegro.

TABLE 6: Boron penetrations for concentration and position in the culms for the studied species submitted to treatment with ruptured diaphragm.

\begin{tabular}{|c|c|c|c|}
\hline \multicolumn{4}{|c|}{ Diafragma rompido } \\
\hline \multicolumn{4}{|c|}{ Dendrocalamus giganteus } \\
\hline Posição & \multicolumn{3}{|c|}{ Penetração (Nota) } \\
\hline Base & \multicolumn{3}{|c|}{$1,77 \mathrm{a}$} \\
\hline Meio & \multicolumn{3}{|c|}{$1,35 \mathrm{~b}$} \\
\hline Topo & \multicolumn{3}{|c|}{$1,11 \mathrm{c}$} \\
\hline \multicolumn{4}{|c|}{ Bambusa vulgaris } \\
\hline Posição & \multicolumn{3}{|c|}{ Penetração (Nota) } \\
\hline Base & \multicolumn{3}{|c|}{$1,87 \mathrm{a}$} \\
\hline Meio & \multicolumn{3}{|c|}{$1,72 \mathrm{a}$} \\
\hline Topo & \multicolumn{3}{|c|}{$1,51 \mathrm{~b}$} \\
\hline \multirow{2}{*}{ Concentração (\%) } & \multicolumn{3}{|c|}{ Posição/Penetração (Nota) } \\
\hline & Base & Meio & Topo \\
\hline 1 & $1,66 \mathrm{Aa}$ & $1,82 \mathrm{Aa}$ & $1,80 \mathrm{Aa}$ \\
\hline 3 & $1,70 \mathrm{Aa}$ & $1,51 \mathrm{Ab}$ & $1,70 \mathrm{Aa}$ \\
\hline
\end{tabular}

Em que: As médias seguidas da mesma letra, maiúscula na horizontal, ou minúscula na vertical, para cada parâmetro, não diferem estatisticamente pelo teste de Tukey $(p>0,05)$.

TABELA 7: Penetrações de cobre para concentração, situação e tempo de tratamento para espécies estudadas. TABLE 7: Copper penetrations for concentration, situation and treatment time for the studied species.

\begin{tabular}{|c|c|c|}
\hline \multirow{3}{*}{ Concentração (\%) } & \multicolumn{2}{|c|}{ Dendrocalamus giganteus } \\
\hline & \multicolumn{2}{|c|}{ Situação/Penetração (Nota) } \\
\hline & Diafragma Íntegro & Diafragma Rompido \\
\hline 1 & $0,80 \mathrm{Ba}$ & $1,23 \mathrm{Ab}$ \\
\hline 3 & $1,02 \mathrm{Ba}$ & $2,40 \mathrm{Aa}$ \\
\hline \multirow{2}{*}{ Tempo (Dias) } & \multicolumn{2}{|c|}{ Dendrocalamus giganteus } \\
\hline & \multicolumn{2}{|c|}{ Penetração (Nota) } \\
\hline 5 & \multicolumn{2}{|c|}{$1,10 \mathrm{~b}$} \\
\hline 10 & \multicolumn{2}{|c|}{$1,63 \mathrm{a}$} \\
\hline 15 & \multicolumn{2}{|c|}{$1,36 \mathrm{ab}$} \\
\hline \multirow[t]{2}{*}{ Concentração (\%) } & \multicolumn{2}{|c|}{ Bambusa vulgaris } \\
\hline & \multicolumn{2}{|c|}{ Penetração (Nota) } \\
\hline 1 & \multicolumn{2}{|c|}{$1,29 \mathrm{~b}$} \\
\hline 3 & \multicolumn{2}{|c|}{$1,87 \mathrm{a}$} \\
\hline
\end{tabular}

Em que: As médias seguidas da mesma letra, maiúscula na horizontal, ou minúscula na vertical, para cada parâmetro, não diferem estatisticamente pelo teste de Tukey $(p>0,05)$. 
TABELA 8: Penetrações de boro para tempo de tratamento, concentração e situação para as espécies estudadas.

TABLE 8: Boron penetrations to treatment time, concentration and situation for the studied species.

\begin{tabular}{|c|c|c|}
\hline Tempo (Dias) & \multicolumn{2}{|c|}{ Dendrocalamus giganteus } \\
\hline 5 & $2,55 \mathrm{Aa}$ & $3,05 \mathrm{Aa}$ \\
\hline 10 & $3,15 \mathrm{Aa}$ & 2,43 Ba \\
\hline 15 & $3,23 \mathrm{Aa}$ & $2,65 \mathrm{Aa}$ \\
\hline \multirow{3}{*}{ Tempo (Dias) } & \multicolumn{2}{|c|}{ Bambusa vulgaris } \\
\hline & \multicolumn{2}{|c|}{ Concentração (\%)/Penetração (Nota) } \\
\hline & 1 & 3 \\
\hline 5 & $3,45 \mathrm{Aa}$ & $3,38 \mathrm{Aa}$ \\
\hline 10 & $3,70 \mathrm{Aa}$ & $2,83 \mathrm{Bb}$ \\
\hline 15 & $3,58 \mathrm{Aa}$ & $3,28 \mathrm{Aab}$ \\
\hline \multirow{3}{*}{ Tempo (Dias) } & \multicolumn{2}{|c|}{ Bambusa vulgaris } \\
\hline & \multicolumn{2}{|c|}{ Situação/Penetração (Nota) } \\
\hline & Diafragma Íntegro & Diafragma Rompido \\
\hline 5 & $3,60 \mathrm{Aa}$ & $3,23 \mathrm{Aa}$ \\
\hline 10 & $3,80 \mathrm{Aa}$ & $2,73 \mathrm{Bb}$ \\
\hline 15 & $3,70 \mathrm{Aa}$ & 3,15 Bab \\
\hline \multirow{3}{*}{ Concentração (\%) } & \multicolumn{2}{|c|}{ Bambusa vulgaris } \\
\hline & \multicolumn{2}{|c|}{ Situação/Penetração (Nota) } \\
\hline & Diafragma Íntegro & Diafragma Rompido \\
\hline 1 & $3,67 \mathrm{Aa}$ & $3,48 \mathrm{Aa}$ \\
\hline 3 & 3,73 Aa & $2,58 \mathrm{Bb}$ \\
\hline
\end{tabular}

Em que: As médias seguidas da mesma letra, maiúscula na horizontal, ou minúscula na vertical, em cada parâmetro, não diferem estatisticamente pelo teste de Tukey $(\mathrm{p}>0,05)$.

o diafragma íntegro ou rompido, para as situações analisadas. Para as situações em que tal diferença foi observada, os colmos com o diafragma íntegro tiveram uma maior penetração.

Esse fato pode ser explicado pela maior difusão do boro ou pela dificuldade encontrada na atribuição das notas por causa da menor nitidez da solução reveladora do boro em relação a do cobre, conforme já observado por Farias Sobrinho et al. (2005), Paes et al. (2005; 2007) e Torres et al. (2011). De modo semelhante, os valores dos demais efeitos apresentados na Tabela 8 , já foram devidamente discutidos anteriormente.

\section{Retenção dos constituintes do CCB}

Na Tabela 9 constam os valores médios da retenção $\left(\mathrm{kg}\right.$ de i.a. $\left.\mathrm{m}^{-3}\right)$ do $\mathrm{CCB}$ nas concentrações de 1 e $3 \%$, para as situações, espécies e tempos de tratamentos testados. Nota-se que as médias de retenção da concentração de 3\% foram superiores em todas as situações testadas. O tratamento preservativo com diafragma rompido obteve as médias mais elevadas de retenção, com um máximo de $19,44 \mathrm{~kg}$ de i.a.m ${ }^{-3}$. Essa retenção, acima das demais, é explicada pela utilização de uma amostra retirada do nó do colmo. Como os nós dos bambus não possuem orientação definida dos vasos, essa desorganização anatômica pode ter prejudicado a ascensão da solução preservativa, proporcionando uma maior concentração nos nós dos colmos, conforme observado por Tiburtino (2012).

Observa-se, de maneira geral, que as médias de retenção do Bambusa vulgaris foram mais elevadas que as do Dendrocalamus giganteus, exceto para o tratamento preservativo com o diafragma rompido. Mesmo assim, pode-se afirmar que, no geral, a quantidade de ingredientes ativos retida nos bambus foi inferior a $6,5 \mathrm{~kg}$ de i.a.m ${ }^{-3}$, valor mínimo recomendado pela NBR 9480 (ABNT, 2009) para a madeira tratada a ser empregada em contato com o solo. 
A estrutura anatômica do bambu pode explicar os baixos valores de retenção observados neste estudo. Como foi discutido anteriormente para a penetração, pois o bambu, ao contrário da madeira, não possui elementos anatômicos dispostos na direção radial, sendo os vasos os

TABELA 9: Retenções do CCB por situação, espécie, tempo de tratamento e concentração da solução preservativa.

TABLE 9: CCB retentions by situation, species, treatment time and concentration of preservative solution.

\begin{tabular}{|c|c|c|c|c|}
\hline \multirow{3}{*}{ Situação } & \multirow{3}{*}{ Espécie } & \multirow{3}{*}{$\begin{array}{c}\text { Tempo } \\
\text { Imunização } \\
\text { (Dias) }\end{array}$} & \multirow{2}{*}{\multicolumn{2}{|c|}{$\frac{\left.\text { Retenção (kg i.a.m }{ }^{-3}\right)}{\text { Concentração da Solução (\%) }}$}} \\
\hline & & & & \\
\hline & & & 1 & 3 \\
\hline \multirow{6}{*}{ Diafragma Íntegro } & \multirow{3}{*}{$\begin{array}{l}\text { Dendrocalamus } \\
\text { giganteus }\end{array}$} & 5 & $2,29 \pm 0,39$ & $1,48 \pm 1,27$ \\
\hline & & 10 & $0,81 \pm 0,12$ & $2,90 \pm 0,23$ \\
\hline & & 15 & $3,20 \pm 1,10$ & $2,40 \pm 1,45$ \\
\hline & \multirow{3}{*}{ Bambusa vulgaris } & 5 & $1,90 \pm 1,43$ & $4,53 \pm 0,83$ \\
\hline & & 10 & $1,00 \pm 0,85$ & $4,21 \pm 0,57$ \\
\hline & & 15 & $4,50 \pm 0,06$ & $7,82 \pm 1,82$ \\
\hline \multirow{6}{*}{ Diafragma Rompido } & \multirow{3}{*}{$\begin{array}{l}\text { Dendrocalamus } \\
\text { giganteus }\end{array}$} & 5 & $1,47 \pm 0,40$ & $4,52 \pm 0,81$ \\
\hline & & 10 & $1,86 \pm 0,30$ & $3,31 \pm 1,32$ \\
\hline & & 15 & $2,20 \pm 1,47$ & $19,44 \pm 8,85$ \\
\hline & \multirow{3}{*}{ Bambusa vulgaris } & 5 & $2,21 \pm 0,50$ & $5,54 \pm 0,40$ \\
\hline & & 10 & $4,00 \pm 0,04$ & $10,52 \pm 0,23$ \\
\hline & & 15 & $5,64 \pm 0,92$ & $7,87 \pm 2,40$ \\
\hline
\end{tabular}

TABELA 10: Retenções de CCB para tempo de tratamento, concentração da solução preservativa e situação para o Dendrocalamus giganteus.

TABLE 10: CCB retentions for treatment time, concentration of preservative solution and situation for Dendrocalamus giganteus species.

\begin{tabular}{ccc}
\hline \multirow{2}{*}{ Tempo (Dias) } & \multicolumn{2}{c}{ Dendrocalamus giganteus } \\
\cline { 2 - 3 } & \multicolumn{2}{c}{ Concentração $(\%) /$ Retenção $\left(\mathrm{kg}\right.$ i.a.m $\left.\mathrm{m}^{-3}\right)$} \\
\cline { 2 - 3 } 5 & $1,88 \mathrm{Aa}$ & 3 \\
\hline 10 & $1,33 \mathrm{Aa}$ & $3,00 \mathrm{Ab}$ \\
15 & $2,70 \mathrm{Ba}$ & $3,11 \mathrm{Ab}$ \\
Tempo (Dias) & \multicolumn{2}{c}{ Situação/Retenção $\left(\mathrm{kg}\right.$ i.a.m $\left.{ }^{-3}\right)$} \\
\hline 5 & Diafragma Íntegro & Diafragma Rompido \\
\cline { 2 - 3 } 10 & $1,88 \mathrm{Aa}$ & $2,99 \mathrm{Ab}$ \\
15 & $1,86 \mathrm{Aa}$ & $2,59 \mathrm{Ab}$ \\
\hline \multirow{2}{*}{ Concentração (\%) } & $2,80 \mathrm{Ba}$ & $10,82 \mathrm{Aa}$ \\
\hline 1 & \multicolumn{2}{c}{ Situação/Retenção (kg i.a.m $\left.{ }^{-3}\right)$} \\
\hline 3 & Diafragma Íntegro & Diafragma Rompido \\
\hline
\end{tabular}

Em que: As médias seguidas da mesma letra, para cada parâmetro, não diferem estatisticamente pelo teste de Tukey $(p>0,05)$. 
principais meios de movimentação dos líquidos no interior dos colmos. Segundo Liese (1985) e Pereira e Beraldo (2008), os vasos ocupam apenas $10 \%$ da área lenhosa do bambu e isto dificulta a difusão da solução preservativa no colmo.

Nas duas espécies de bambus e nas duas concentrações adotadas, o tratamento preservativo com diafragma rompido exibiu os valores mais elevados de retenção do CCB. Para o Dendrocalamus giganteus, houve significância estatística entre as interações tempo x concentração; tempo x situação de tratamento (diafragma íntegro ou rompido); e concentração x situação de tratamento. Já para o Bambusa vulgaris, resultados significativos foram obtidos para o parâmetro concentração e para a interação tempo x situação de tratamento.

$\mathrm{Na}$ Tabela 10 constam as comparações entre médias das retenções em colmos de Dendrocalamus giganteus. Apenas o tempo de 15 dias causou diferença entre as médias, tendo na concentração de $3 \%$ sido verificado o maior valor de retenção. Apenas para esta retenção, os tempos de tratamento testados causaram diferenças entre as médias, tendo o tempo de 15 dias promovido uma retenção superior aos demais tempos de tratamento testados. Resultado semelhante foi observado quanto ao efeito do diafragma, em que apenas para os colmos submetidos ao tratamento preservativo durante 15 dias, aqueles com o diafragma rompido tiveram uma maior retenção. Para este caso, o tempo de 15 dias também causou uma retenção superior aos demais tempos testados.

Quanto ao efeito da concentração da solução preservativa sobre a situação de tratamento (diafragma íntegro ou rompido) observa-se que a concentração de $1 \%$ não revelou diferença significativa entre as situações, no entanto a de $3 \%$ causou maior valor de retenção nos colmos tratados com o diafragma rompido. Para este caso, a concentração de $3 \%$ proporcionou uma maior média de retenção quando comparada com a de $1 \%$.

Observa-se para o Bambusa vulgaris (Tabela 11) que a concentração de $3 \%$ proporcionou uma maior retenção. Nota-se também que o tempo de tratamento de 10 dias proporcionou uma maior retenção nos colmos tratados com o diafragma rompido. Para os demais tempos de tratamento, não houve diferenças significativas entre a situação de tratamento (diafragma íntegro ou rompido). $\mathrm{O}$ tempo de 15 dias proporcionou uma maior retenção nos colmos tratados com o diafragma íntegro, enquanto para aqueles tratados com o diafragma rompido, os tempos de 10 e 15 dias proporcionaram retenções superiores aos tratados durante 5 dias.

De modo geral, o rompimento do diafragma dos colmos das espécies testadas causou aumento na retenção do CCB. Como já afirmado anteriormente, os diafragmas dificultam a movimentação da solução preservativa no sentido longitudinal dos colmos.

Ao contrário das madeiras, como o bambu não possui raios, a transpiração ocorre somente pelos vasos, apenas 10\% no colmo (LIESE, 1985; PEREIRA; BERALDO, 2008). Além disto, a impermeabilidade das camadas internas e externas nos colmos é outro fator importante que impede a transpiração na direção transversal, desta forma

TABELA 11: Retenções de CCB para tempo, situação de tratamento e concentração da solução preservativa para o Bambusa vulgaris.

TABLE 11: CCB retentions for time, treatment situation and concentration of preservative solution for Bambusa vulgaris species.

\begin{tabular}{ccc}
\hline \multirow{2}{*}{ Tempo (Dias) } & \multicolumn{2}{c}{ Bambusa vulgaris } \\
\cline { 2 - 3 } & \multicolumn{2}{c}{ Tratamento/Retenção CCB (kg i.a.m ${ }^{-3}$ ) } \\
\cline { 2 - 3 } & Diafragma Íntegro & Diafragma Rompido \\
\hline 5 & $3,21 \mathrm{Ab}$ & $3,87 \mathrm{Ab}$ \\
10 & $2,60 \mathrm{Bb}$ & $7,25 \mathrm{Aa}$ \\
15 & $6,17 \mathrm{Aa}$ & $6,76 \mathrm{Aa}$ \\
\hline Concentração (\%) & \multicolumn{2}{c}{ Retenção CCB (kg i.a.m $\left.{ }^{-3}\right)$} \\
\hline 1 & \multicolumn{2}{c}{$3,20 \mathrm{~b}$} \\
\hline
\end{tabular}

Em que: As médias seguidas da mesma letra, maiúscula na horizontal, ou minúscula na vertical, em cada parâmetro, não diferem estatisticamente pelo teste de Tukey $(p>0,05)$. 
TABELA 11: Retenções de CCB para tempo, situação de tratamento e concentração da solução preservativa para o Bambusa vulgaris.

TABLE 11: CCB retentions for time, treatment situation and concentration of preservative solution for Bambusa vulgaris species.

\begin{tabular}{|c|c|c|}
\hline \multirow{3}{*}{ Tempo (Dias) } & \multicolumn{2}{|c|}{ Bambusa vulgaris } \\
\hline & \multicolumn{2}{|c|}{ Tratamento/Retenção CCB (kg i.a.ms) } \\
\hline & Diafragma Íntegro & Diafragma Rompido \\
\hline 5 & $3,21 \mathrm{Ab}$ & $3,87 \mathrm{Ab}$ \\
\hline 10 & $2,60 \mathrm{Bb}$ & $7,25 \mathrm{Aa}$ \\
\hline 15 & $6,17 \mathrm{Aa}$ & $6,76 \mathrm{Aa}$ \\
\hline Concentração (\%) & \multicolumn{2}{|c|}{ Retenção CCB (kg i.a.mº } \\
\hline 1 & \multicolumn{2}{|c|}{$3,20 \mathrm{~b}$} \\
\hline 3 & \multicolumn{2}{|c|}{$6,75 \mathrm{a}$} \\
\hline
\end{tabular}

Em que: As médias seguidas da mesma letra, maiúscula na horizontal, ou minúscula na vertical, em cada parâmetro, não diferem estatisticamente pelo teste de Tukey $(p>0,05)$.

o único local para ocorrer esse processo são as extremidades dos colmos.

\section{CONCLUSÕES}

Os colmos do Dendrocalamus giganteus submetidos ao processo com o diafragma íntegro absorveram um maior volume de solução de CCB para ambas as concentrações testadas, enquanto para o Bambusa vulgaris as maiores absorções ocorreram com o diafragma rompido.

Os valores da penetração de cobre e boro nos colmos para ambas as condições de tratamento e espécies de bambu foram baixos, sendo caracterizados entre penetração vascular e penetração parcial periférica. Entre as três regiões analisadas verificou-se que houve maior penetração nos colmos na posição da base.

A combinação de 15 dias de tratamento com $3 \%$ de ingredientes ativos de CCB proporcionou melhor média de penetração e retenção da solução para as situações e espécies empregadas.

As retenções dos ingredientes ativos do CCB obtidas nas duas condições de tratamento, para ambas as espécies de bambu testadas, salvo algumas exceções, foram abaixo do recomendado pelas normas brasileiras para o tratamento preservativo de madeira.

De modo geral, houve pouca movimentação da solução preservativa ao longo dos colmos de ambas as espécies testadas, sendo melhorada com o rompimento do diafragma.

\section{AGRADECIMENTOS}

À Fundação de Amparo à Pesquisa e Inovação do Espírito Santo - FAPES pela concessão de bolsa de estudo a Rogy Frigeri Tiburtino, à Montana Química pela doação do produto "MOQ OX 50" e ao Sr. Sebastião Ferreira, pela doação dos bambus.

\section{REFERÊNCIAS BIBLIOGRÁFICAS}

ASSOCIAÇÃO BRASILEIRA DE NORMAS TÉCNICAS. NBR 9480: peças roliças preservadas de eucalipto para construções rurais: requisitos. Rio de Janeiro, 2009. 12p.

ASSOCIAÇÃO BRASILEIRA DE NORMAS TÉCNICAS. NBR 6232: penetração e retenção de preservativos em postes de madeira. Rio de Janeiro, 2013. 16p.

AZZINI, A.; BERALDO, A.L. Métodos práticos para utilização do bambu. Campinas: UNICAMP, 2001. 14p.

BERALDO, A.L.; AZZINI, A. Bambu: características e aplicações. Guaíba: Agropecuária, 2004. $128 \mathrm{p}$.

DA SILVA. O.F. Estudo sobre a substituição do aço liso pelo Bambusa vulgaris, como reforço de vigas de concreto, para o uso em construções rurais. 2007. 141f. Dissertação (Mestrado em Engenharia Civil) - Universidade Federal de Alagoas, Maceió, 2007.

FARIAS SOBRINHO, D.W.; PAES, J.B.; 
FURTADO, D.A. Tratamento preservativo da madeira de algaroba (Prosopis juliflora (Sw.) D.C.), pelo método de substituição de seiva. Cerne, Lavras, v. 11, n. 3, p. 225-236, 2005.

FERREIRA, L.G.R. Qualidade do tratamento preservativo do bambu. 2010. 29f. Monografia (Engenharia Florestal) - Universidade Federal do Espírito Santo, 2010.

HUI, C.; YANG, Y. China's bamboo: culture, resources, cultivation, utilization. 33. ed. Beijing: International Network for Bamboo and Rattan, 2010, 224p.

LIESE, W. Bamboos: biology, silvics, properties, utilization. Eschborn: Gesellschaft für Technische Zusammenarbeit - GTZ, 1985. 132p.

NATIONAL MISSION ON BAMBOO APPLICATIONS - NMBA. Preservation of bamboo. Disponível em: <http:// bambootech.org/ files/ PRESERVATION \%20BOOK.pdf $>$. Acesso em: 01 maio 2012.

PAES, J.B.; MORESCHI, J.C.; LELLES, J.G. Avaliação do tratamento preservativo de moirões de Eucalyptus viminalis Lab. e de bracatinga (Mimosa scabrella Benth.) pelo método de substituição da seiva. Ciência Florestal, Santa Maria, v. 15, n. 1, p.75-86, 2005.

PAES, J.B.; et al. Tratamento preservativo de peças roliças de leucena (Leucaena leucocephala (Lam.) de Wit.) pelo método de substituição da seiva. Revista Ciências Agrárias, Belém, n. 47, p. 231-246, 2007.

PAES, J.B.; et al. Caracterização físico-mecânica do laminado colado de bambu. Ciência Florestal, Santa Maria, v.19, n.1, p. 41-51, 2009.

PEREIRA, M.A.R; BERALDO, A.L. Bambu de corpo e alma. Bauru: Canal 6, 2008. 240p.

SALES-CAMPOS, C.; VIANEZ, B.F.; MENDONÇA, M.S. Estudo da variabilidade da retenção do preservante CCA tipo A na madeira de Brosimum rubescens Taub. Moraceae - (paurainha) uma espécie madeireira da Região Amazônica. Revista Árvore, Viçosa, v.27, n.6, p.845-853, 2003.

SANTOS, R.L.; LOPES, J.D.S. Construções com bambu: opção de baixo custo. Viçosa: Centro de Produções Técnica, 1998. 40p. (Série Construções Rurais, 160).

STEEL, R.G.D.; TORRIE, J.H. Principles and procedures of statistic: a biometrical approach. 2 ed. New York: Mc-Graw Hill, 1980. 633 p.

TIBURTINO, R.F. Qualidade e eficiência do tratamento preservativo de duas espécies de bambu. 2012. 94f. Dissertação (Mestrado em Ciências Florestais) - Universidade Federal do Espírito Santo, Jerônimo Monteiro, 2012.

TORRES, P. M. A. et al. Tratamento preservativo da madeira juvenil de Eucalyptus camaldulensis Dehnh. pelo método de substituição de seiva.

Cerne, Lavras, v.17, n.2, p.275-282, 2011. 